a precied edition of any standard undergraduate medical textbook. If the problem confronting the young inexperienced house physician is to be approached realistically the discussion should resolve itself around the main presenting symptoms of the patient, i.e. coma, acute chest pain, acute abdominal pain, acute dyspnoea, etc. The differential diagnosis of each symptom could then be discussed giving the salient features which should lead the doctor to one as opposed to the other causes of the symptom. At present the book assumes that the correct diagnosis has been at least considered.

\section{Introduction to Clinical Neurology}

By Sir Gordon Holmes. Revised by Bryan Matthews. Third edition. Pp. 187. Edinburgh and London: E. \& S. Livingstone, 1968. $£ 115 \mathrm{~s}$.

It has been a pleasure to re-read this classical text and the publishers must be commended and Dr Matthews congratulated on their new edition. Thanks to them we still have this best of introductions to the art of Neurology. Most of the text remains the master's, but Dr Matthews has made alterations and additions, notably a rather too short chapter on 'Neurology in Children'. At times one wishes that the alterations were more readily identifiable although in other places they are betrayed by differences in style. Perhaps subsequent editions might make the emendations more obvious and record Sir Gordon Holmes' views in a footnote or appendix. The book is not perfect, but very few books are and no-one can be abreast of every development. Brouwer's concept of the eye muscle nuclei, for example, has long been rendered obsolete by the studies of Professor Warwick. The neophyte wishing to test taste with the terminals of a torch battery must choose a weapon which actually has terminals, otherwise he should make his own electrical taste tester or try to follow the instructions of Sir Gordon Holmes and apply sugar, salt, quinine, citric acid in powder to the anterior and posterior (sic) parts of the tongue. It is amusing to see the caudate nucleus still omitted from the legend of Figure 6 or 7 , but these are minor blemishes in a valuable and interesting book. 